\title{
Design and Implementation of Smart Home using Cisco Packet Tracer Simulator 7.2
}

\author{
Pitcheri Praveen Kumar, Murali Krishna, M,R Ramprakash
}

\begin{abstract}
In the new time of technology improvement today, without understanding the new time of innovation has turned into a need and that is regularly utilized for everyday existence of the present society. Where nearly in the greater part of the regions have had the option to feel from the advancement of new time of such innovation, as in the field of PC systems administration and hardware. For this situation, the requirement for actualizing the home innovation is progressively required to make a smart house, where during this time the home control is finished by executing the gadgets. Home can be mechanized through the turn on or off switch utilizing these hardware gadgets. The planning of Smart Home is an application which is a mix of innovation and administrations gave to the home condition with explicit capacities meant to improve the security, proficiency, and furthermore solace of its occupants. In the planning some portion of keen home framework normally comprises of control devices,monitoring apparatuses and programmed there are a few gadgets that can be gotten to utilizing an advanced mobile phone or PC which is associated with the Internet organize.

IOT (Internet of things) is where a few things can be associated together,sensed and furthermore remotely controlled over the system. This paper manages the execution of brilliant home utilizing using new released version of Cisco packet tracer simulator 7.2, because this feature include different kind of sensor and actuator and also having different types of smart device used for home automation.
\end{abstract}

Keywords:IOE,Sensor,Smart home,packet tracer 7.2 simulator.

\section{INTRODUCTION}

The IOT(internet of things) is a system which is equipped for interfacing everything to the web through remote sensor networks.IOT(internet of things) is made out of two words :web and things. The IOT innovation usage made a change in new developments in the fields of horticulture, industry and vitality dispersion by including the significant data with the assistance of various kinds of sensors. As per Cisco organizing there are enormous number of organizations and research associations which gives the effects of Internet of things on the web and the economy in the following five to ten years.

The internet of things was first presented by pioneer Kevin Ashton in the time of 1999 to characterize that the items in the physical globe can be utilized to associated with

Revised Manuscript Received on September 10, 2019

Pitcheri Praveen Kumar, Department Of Electronics and Communications Engineering, Anurag Group of Institutions, Hyderabad, Telangana, India.

(Email: pitcheripraveenreddy@gmail.com)

Murali Krishna, Department Of Electronics and Communications Engineering, Anurag Group of Institutions, Hyderabad, Telangana, India. (Email: muralikrishnaece@cvsr.ac.in)

M,R Ramprakash, Department Of Electronics and Communications Engineering, Anurag Group of Institutions, Hyderabad, Telangana, India.

(Email: ramprakashece@cvsr.ac.in) the web by utilizing of the sensors. He gave that the capacity of interfacing Radio recurrence Identification(RFID) utilized in business to the web to follow the different kinds of products/materials prepared selling without prerequisite of the human intercession.

The present internet of things(IOT) has a capacity to depict the various sorts articles, gadgets and sensors to interface with the web. As a result,IOT is commonly new idea, yet coordinating systems and PCs to oversee and control things had existed in this genuine world for around quite a few years.

Internet of things (IOT) enables items and clients to speak with one another by utilizing of a novel IP address to each article to distinguish which clients are going to getting to what asset of the system effectively. IOT additionally depicts a universe of system wherein all items are associated with the system so information can be partaken in a system. Everyone as of now has an advanced cell, however a telephone isn't savvy rather it encourages its client to make more intelligent decisions.also depicts a universe of system wherein each article is associated with the system so information can be shared. Everybody as of now has an advanced cell, however a telephone is certainly not a brilliant as opposed to it causes its client to settle on more astute choices.

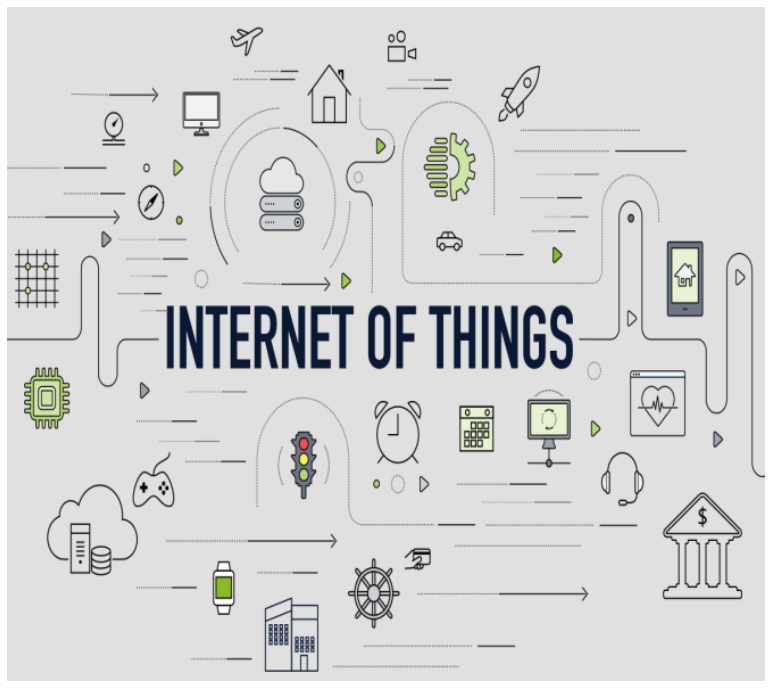

FIG -1.1. Internet of Things

\section{RELATED WORK}

Smart home is a living home that comprise of shrewd item to improve the every home action ahead of time, that can be mechanizing exercises of the home without the contribution of client such checking home condition should

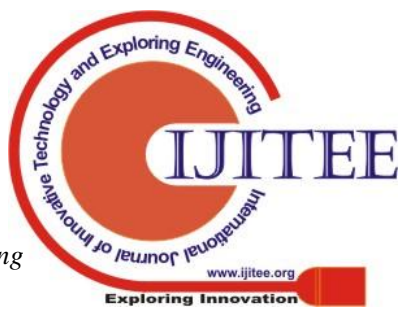


be possible by utilizing different sensor (Temperature, Humidity, sound, smoke, twist) at that point ventilate the

earth dependent on the data of sensor..

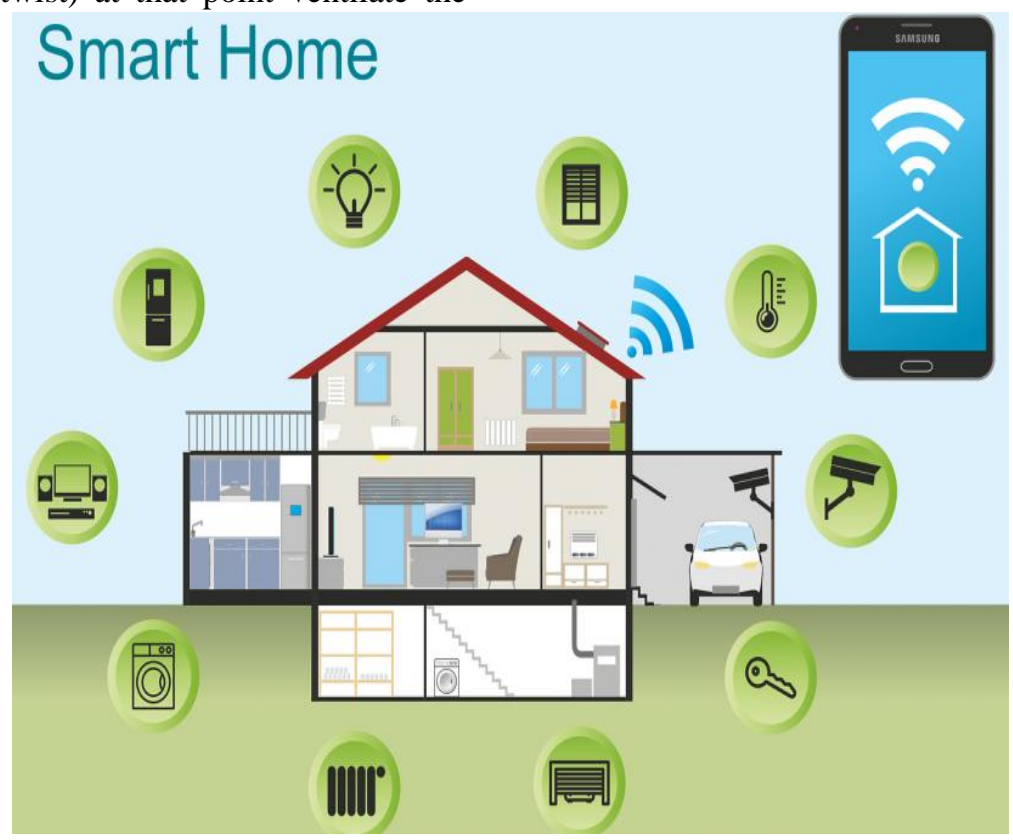

FIG -2.1. Smart home with IOT

To execute smart home, Cisco packet tracer 7.2 variant is utilized which is another discharged innovation that incorporates every smart article planned for office mechanization. These gadgets are: brilliant fan, keen light, savvy window, shrewd entryway . Be that as it may, the home door gives the controlling components by enlisting every single smart gadget separately by means of the cloud (WAN).

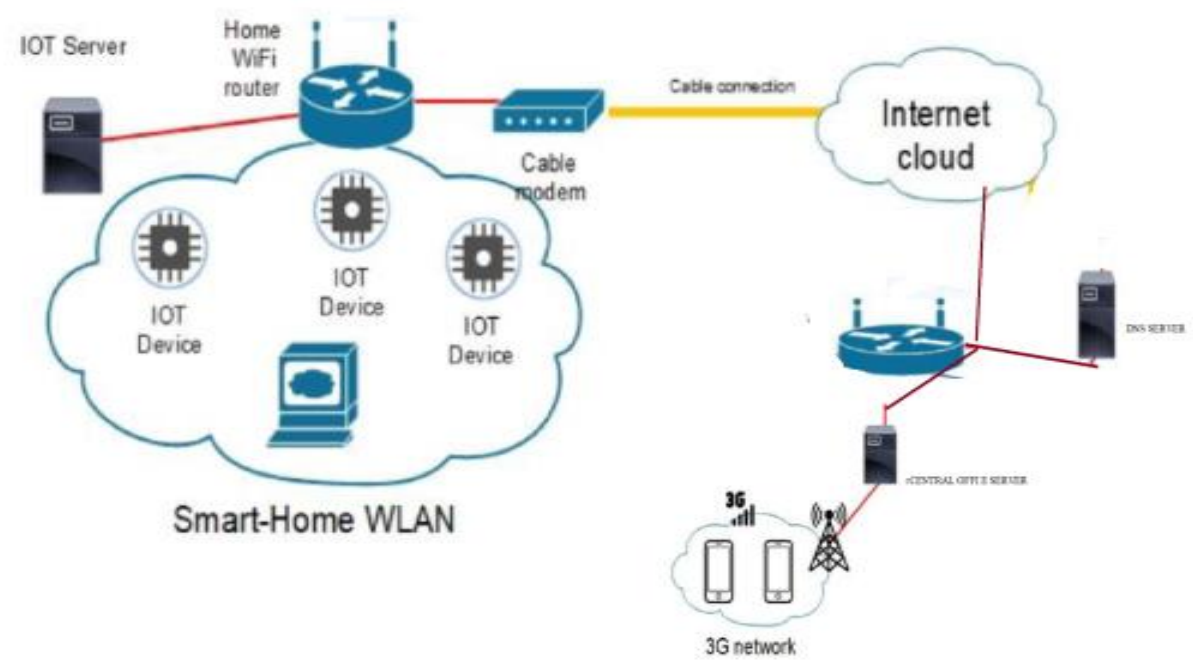

FIG-2.2 Block diagram of Smart Home Automation 


\section{METHODOLOGY}

The circuit chart of keen home Automation is given as beneath appeared

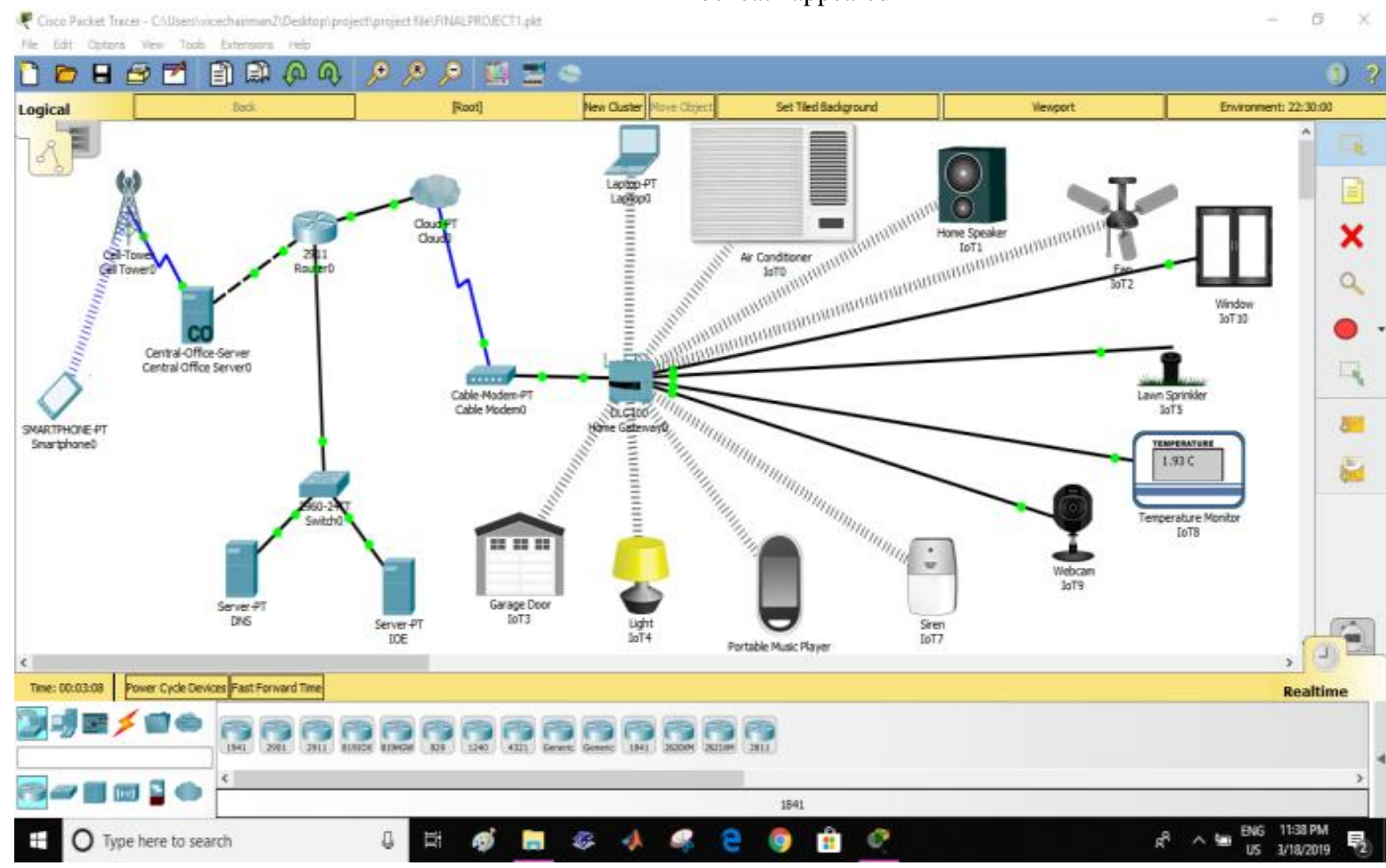

FIG-3.1 Smart home circuit diagram

As clear at the above figure the house is created by utilizing the system test system which comprises of various gadgets an IOT passage with associated brilliant gadgets, IOT server, DNS server, IOT cloud (WAN), cell tower, Central office server, ISP server, advanced cell ,link modem and PC.

The server contains 1 . internet of things server 2.Domain name server.The IOT server stores all the recognized data from the working environment and give customers an endorsed access to the advantages by entering username and mystery state.

\subsection{Algorithm}

The Algorithm is explained as follows

Step 1: Start the project.

Step 2: Open the pkt file and save the file.

Step 3: Add the required components to the work space as packet tracer simultor shown above.

Step 4: Connect all devices in work space Using cables.

Step 5 : Configure the device and setup internet service provider router.

Step 6: Add Home Gateway to the Network.

Step 7 : Connect smart Devices to the Wireless Network.

Step 8: Add End User Device to the Network

Step 9: Stop

\subsection{Flow Chart}

The flow chart is explained as follows

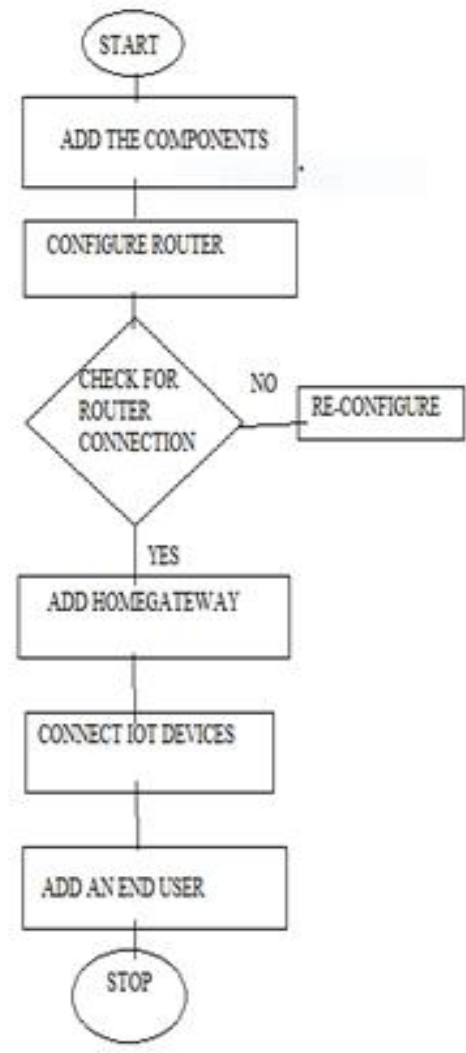

FIG-3.2 Flow chart 


\section{IMPLEMENTATION \& RESULTS}

\subsection{Device Configurations}

Assigning host name and IP address for main router Router>en

Router\#config te

Router(config)\#hostname main

main(config)\#int g0/0

main(config-if)\#ip add 192.168.1.1

255.255.255.0

main(config-if)\#no shutdown

main(config)\#int g0/1

main(config-if)\#ip add 192.168.2.1

255.255.255.0

main(config-if)\#no shut

main(config)\#int g 0/2

main(config-if)\#ip add 192.168.3.1

255.255.255.0

main(config-if)\#no shutdown

Configuration of dhcp server for cell and IOE device main(config)\#ip dhcp excluded-address

192.168.2.1 192.168.2.13

SP(config)\#ip dhcp pool <name >

main(dhcp-config)\#network 192.168.2.0

255.255.255.0

main(config)\#default-router 192.168.2.1

main(dhcp-config)\#dns-server 192.168.1.11

main(config)\#ipdhcp excluded-address

192.168.3.1 192.168.3.11

main(config)\#ipdhcp pool <pool name>

main(dhcp-config)\#network 192.168.3.0

255.255.255.255

main(config)\#default-router192.168.3.1

main(dhcp-config)\#dns-server 192.168.1.11

main(dhcp-config)\#exit

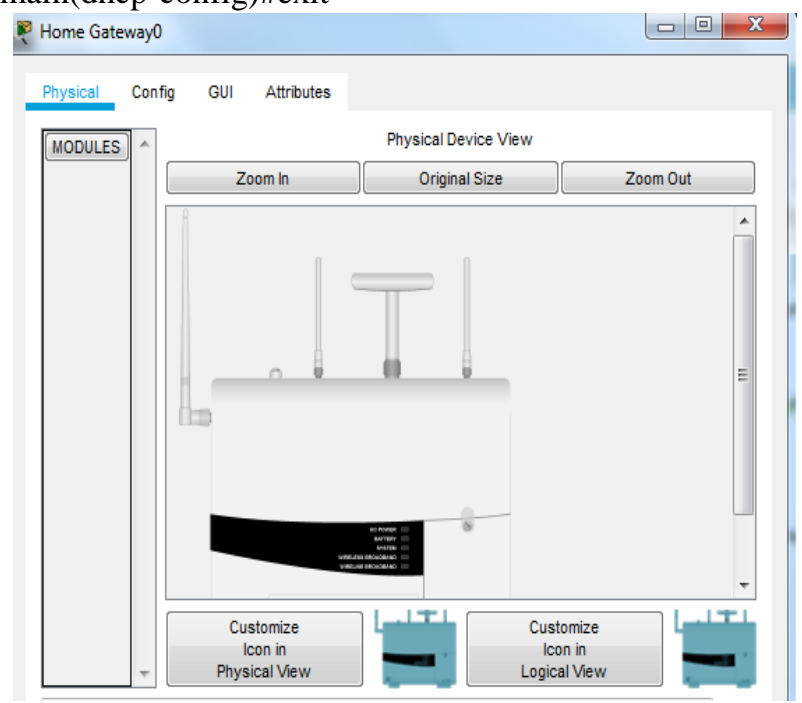

FIG-4.1 home gateway

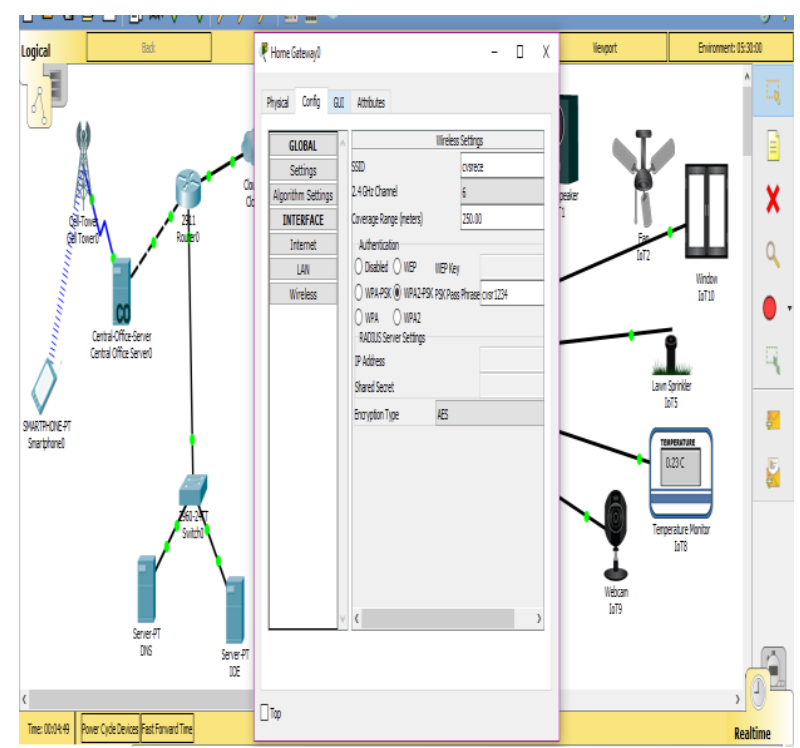

FIG-4.2 Connecting the wireless devices to homegate way

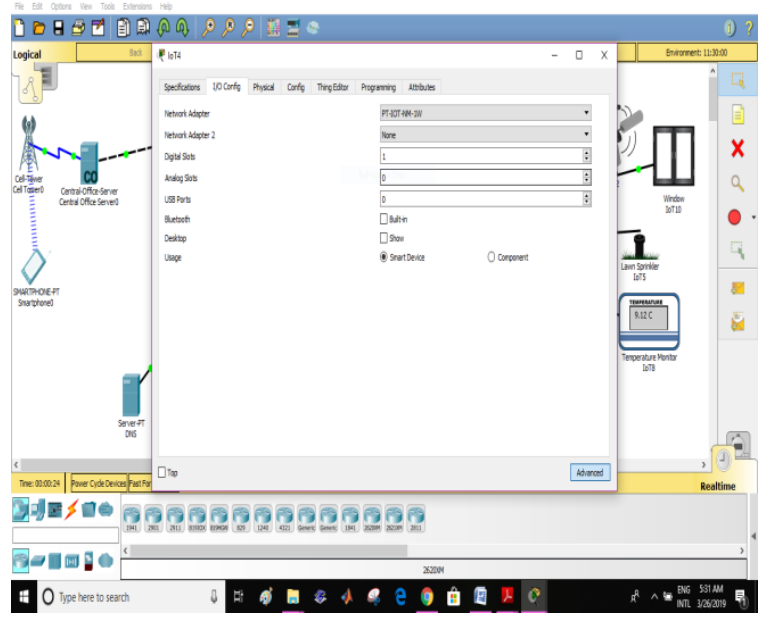

FIG-4.3 Changing devices to wireless module

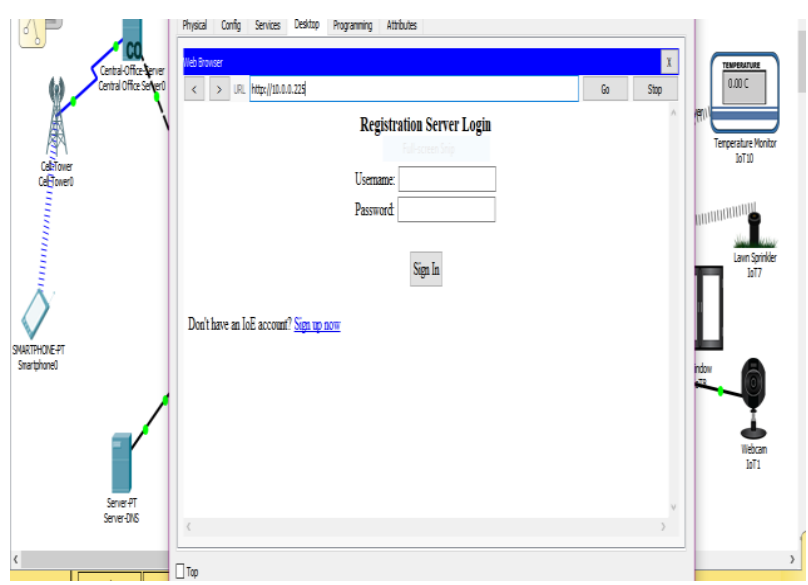

FIG 4.4 Creating an account to register devices.

\subsection{IOT-Layouts.}

IoT rationale availability was set up on the highest point of the system network. As appeared in the Figure all IoT gadgets must be set to be associated remotely to the IoT server utilizing recently made username, secret phrase and the server IP.

Published By:

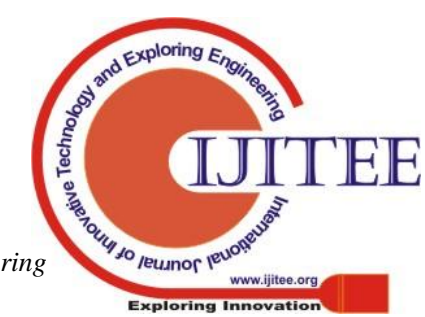


Effective network was set up when "Interface" catch changed into "Invigorate". All gadget must utilize the equivalent IoT qualifications, same certifications were likewise utilized by the mortgage holder for passing the verification when interfacing by means of program to the principle IoT checking landing page as appeared in the Figure As the IoT server was arrangement likewise for DNS administrations, the iothomepage.com was interpreted with the IoT server static IP. When client is associated with IoThomepage.com it is conceivable to picture the status of the IoT gadgets yet it is additionally conceivable to audit the collaboration rationale between them. As noticeable in the Figure in this activity f IoT keen gadgets were utilized: movement indicator, alarm, carport entryway, fan and smoke alarm however these were just for impact the ecological variable of the reenactment

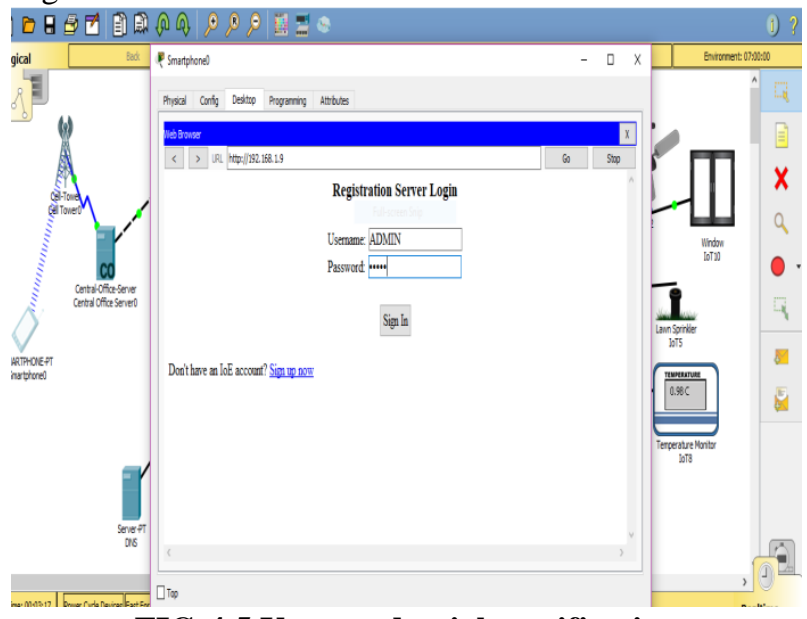

FIG-4.5 User credentials verification

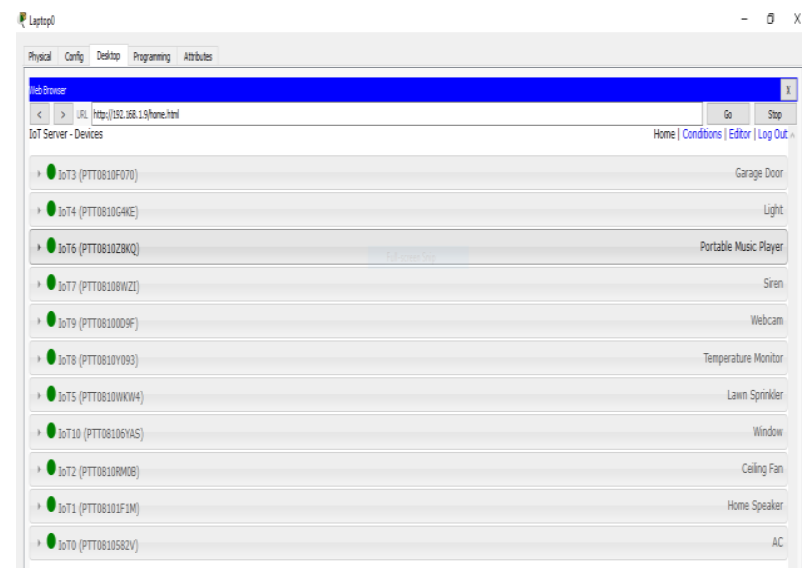

FIG-4.6 list of devices registered in server

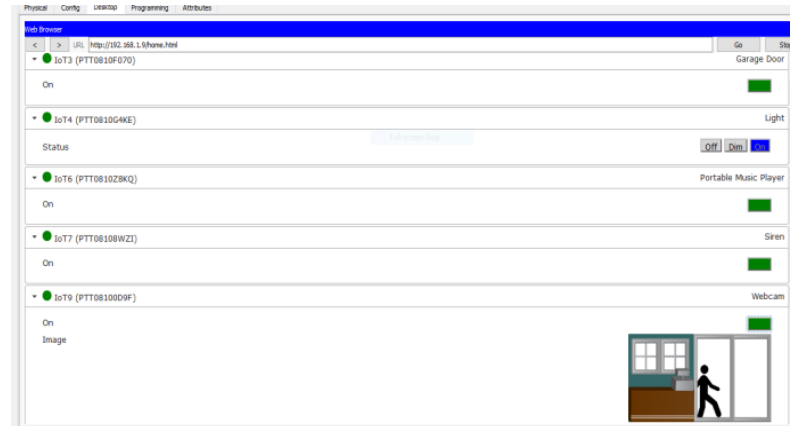

FIG-4.7 Devices are in on state when operated through laptop.
The protocols used for the Network are shown as follows.

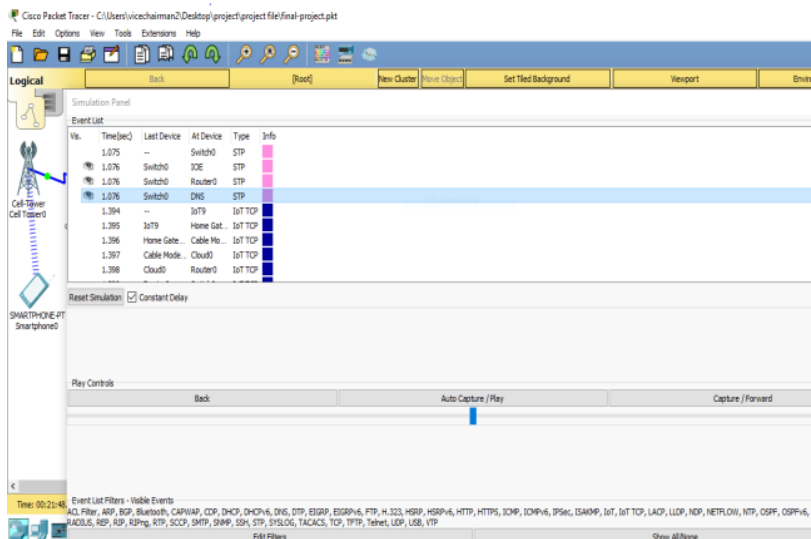

FIG-4.8 Simulation environment in transmitting mode used protocol

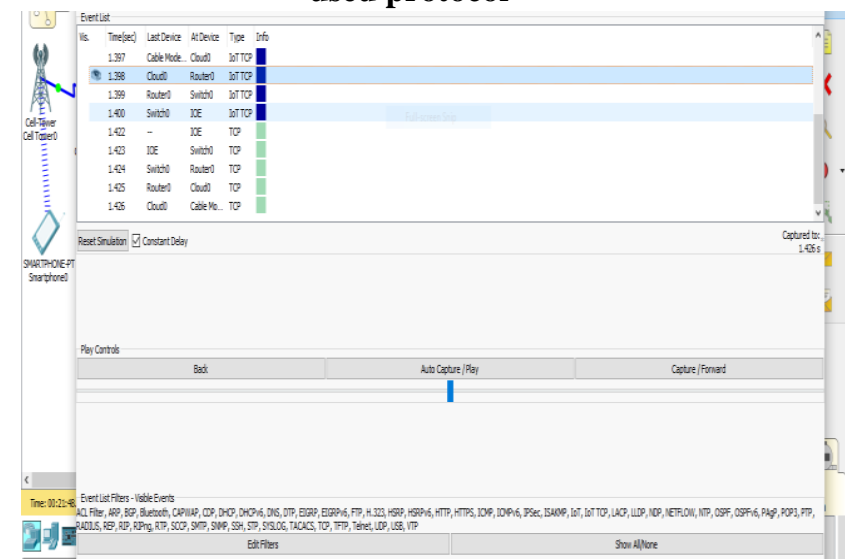

FIG-4.9 Simulation environment in receving mode used protocol

\section{CONCLUSION}

This venture work is to examine the idea of the Internet of things and its pertinence in home robotization setting. Internet of things is another innovation that is utilized for the interconnection of the gadgets with the assistance of the web association.

It empowers the gadgets to detect and screen gadgets remotely. It has been told the best way to effectively manufacture a keen home that will contain advanced gadgets to thusly screen and control every action and occasions inside home utilizing IoT shrewd gadgets. So as to demonstrate the achievability of the work a reenactment instrument is utilized for planning a keen home

\section{REFERENCES}

1. "The Internet of Things in an Enterprise Context " in Future Internet-FIS 2008 Lecture Notes in Computer Science Vol. 54682009

2. Smart home system based on iot technologies." Computational and Information Sciences (ICCIS), 2013 Fifth International Conference on. IEEE

3. http://www.packettracernetwork.com/

4. Pike research on smart cities [online] Available: http://www.pikeresearch.com/research/smart-cities.

5. S. Hedetniemi, A. Liestman, "A survey of gossiping and broadcasting in communication networks,"

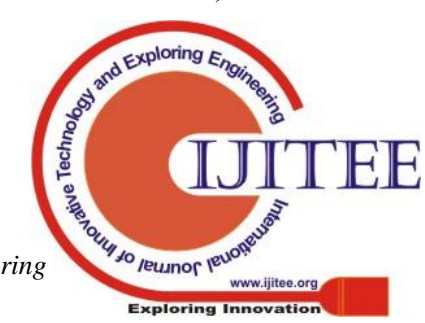

